SubmitTed TO ApJ

Preprint typeset using LATEX style emulateapj v. 19/02/01

\title{
CONTINUUM EMISSION BY COOLING CLOUDS
}

\author{
MARK DIJKSTRA \\ Harvard-Smithsonian Center for Astrophysics, 60 Garden Street, Cambridge, MA 02138, USA \\ Submitted to ApJ
}

\begin{abstract}
The collapse of baryons into the center of a host dark matter halo is accompanied by radiation that may be detectable as compact ( $\lesssim 10 \mathrm{kpc}$ ) UV-continuum and Ly $\alpha$ emission with Ly $\alpha$ luminosities as high as $\sim 10^{42}-10^{43}$ $\mathrm{erg} \mathrm{s}^{-1}$ in halos of mass $M=10^{11}-10^{12} M_{\odot}$. We show that the observed equivalent width (EW) of the Ly $\alpha$ line emitted by these cooling clouds is EW $\lesssim 400 \AA$ (restframe). These luminosities and EWs are comparable to those detected in narrowband surveys for redshifted Ly $\alpha$ emission. The rest-frame ultraviolet of Ly $\alpha$ emitting cooling clouds radiation may be dominated by two-photon transitions from $2 \mathrm{~s} \rightarrow 1 \mathrm{~s}$. The resulting spectrum can distinguish cooling clouds from a broad class of young star forming galaxies.
\end{abstract}

Subject headings: cosmology: theory-galaxies: high redshift-galaxies: formation-radiation mechanisms: general

\section{INTRODUCTION}

Gas collapse into the center of a host dark matter halo requires the release of gravitational binding energy in the form of cooling radiation. The efficiency of gas cooling therefore plays an important role in the formation of stars and galaxies. For example, it has been shown that trapping of cooling radiation can affect the fragmentation of a collapsing gas cloud (Spaans \& Silk 2006). Understanding cooling radiation is therefore essential in our understanding of galaxy formation. A large fraction of cooling radiation may emerge as Ly $\alpha$ emission (Haiman et al. 2000; Fardal et al. 2001), possibly accompanied by emission from helium (Yang et al. 2006). Galaxies may therefore be visible as extended Ly $\alpha$ sources before star formation occurs. This realization coincided with the discovery of two large Ly $\alpha$ 'blobs' (LABs) by Steidel et al. (2000)]. Naturally, LABs have been associated with cooling radiation.

Since the discovery of LABs by Steidel et al. (2000) several tens of new LABs have been discovered (Matsuda et al. 2004; Dey et al. 2005; Saito et al. 2006; Ouchi et al. 2008b). Various groups have investigated what powers Ly $\alpha$ emission in LABs (e.g. Matsuda et al. 2006; Saito et al. 2007). Recent studies suggest that the majority is likely powered by (obscured) stars and/or AGN (e.g. Basu-Zych \& Scharf 2004; Matsuda et al. 2007), or by a superwind (e.g. Taniguchi \& Shioya 2000; Mori et al. 2004). However, in some LABs it is possible to rule out all these mechanisms, as each is expected to produce characteristic metal lines at flux levels that are not detected. This has led to the conclusion that cooling radiation may have been detected (Nilsson et al. 2006; Saito et al. 2007; Smith \& Jarvis 2007).

Cooling radiation may also manifest itself as compact Ly $\alpha$ emission (rather than spatially extended) with luminosities comparable to those observed in high-redshift Ly $\alpha$ emitting galaxies. Haiman et al. (2000) pointed out that $\sim 30 \%$ of their total computed cooling luminosity may originate from the central $\lesssim 10 \%$ in radius. Furthermore, Fardal et al. (2001) and Yang et al. (2006) found that spatially extended cooling gas clouds in 3-D simulations contain dense compact clumps $(<10 \mathrm{kpc}$, $n_{\mathrm{H}, \max } \sim 50 \mathrm{~cm}^{-3}$ ) which dominate the total Ly $\alpha$ cooling emis-

1 Extended Ly $\alpha$ emission is quite common around radio galaxies, and is thought to be powered by some sort of jet-IGM interaction (e.g. Chambers et al. 1990; Villar-Martín 2007). However, the LABs discovered by Steidel et al. (2000) have no associated radio-galaxies. sion. Since it is plausible that star formation takes place inside these densest clumps, a major challenge lies in determining which process provides the biggest contribution to the Ly $\alpha$ luminosity emerging from clumps: cooling or young stars (e.g. Kereš et al. 2005, for a more detailed discussion).

Dijkstra et al. (2006) quantified the properties of cooling radiation by performing Monte-Carlo Ly $\alpha$ radiative transfer calculations in collapsing optically thick gas clouds, and found that Ly $\alpha$ cooling radiation should be systematically blueshifted. In practice it may prove difficult to actually observe this blueshift, as this requires the detection of other lines, which are often absent. Dijkstra et al. (2006) also showed that Ly $\alpha$ cooling radiation may reveal itself through a frequency dependent surface brightness profile: the surface brightness profile of the reddest Ly $\alpha$ photons is expected to be flatter than that of the bluest Ly $\alpha$ photons. However, the strength of this signal is model dependent and may be too subtle to be detectable. Therefore, it is desirable to have more diagnostics that characterize cooling radiation.

The main goal of this paper is to provide new diagnostics to identify (compact) cooling clouds in data generated by existing and upcoming telescopes. We compute the continuum emission of cooling clouds redward of Ly $\alpha$, and show that this is dominated by two-photon transitions from $2 s \rightarrow 1$ s. The spectrum of this continuum has a peculiar shape which may distinguish cooling clouds from young star forming galaxies.

The outline of this paper is as follows. In $\S 2$ we illustrate that cooling radiation may be detectable as compact Ly $\alpha$ emission. This is followed by a calculation of the spectrum of cooling radiation at wavelengths redward of Ly $\alpha$ in $\S 3$. Finally, in $\S 4$ we discuss the results of this paper, and present our main conclusions.

2 The term 'Ly $\alpha$ cooling radiation' that is used throughout this paper refers to both real (i.e. collisionally excited) cooling radiation, as well as Ly $\alpha$ radiation that is emitted as a by-product of recombination cooling (even though Ly $\alpha$ radiation that is produced in radiative cascades following recombination is technically not cooling radiation, since it does not transport any heat from the gas). In both cases, the Ly $\alpha$ radiation is emitted in a 'proto-galactic' gas cloud that is still collapsing to from stars. Furthermore, for neutral fractions as low as $x_{\mathrm{HI}}=10^{-4}$, the 'real' Ly $\alpha$ cooling rate due to collisional excitation, $\Lambda_{\mathrm{Ly} \alpha}$ is equal to the $\operatorname{Ly} \alpha$ emission rate due to recombination (for $T_{\text {gas }}=3 \times 10^{4} \mathrm{~K}$, see Appendix B. Hence, even recombining H II regions can emit a significant amount of Ly $\alpha$ cooling radiation. 


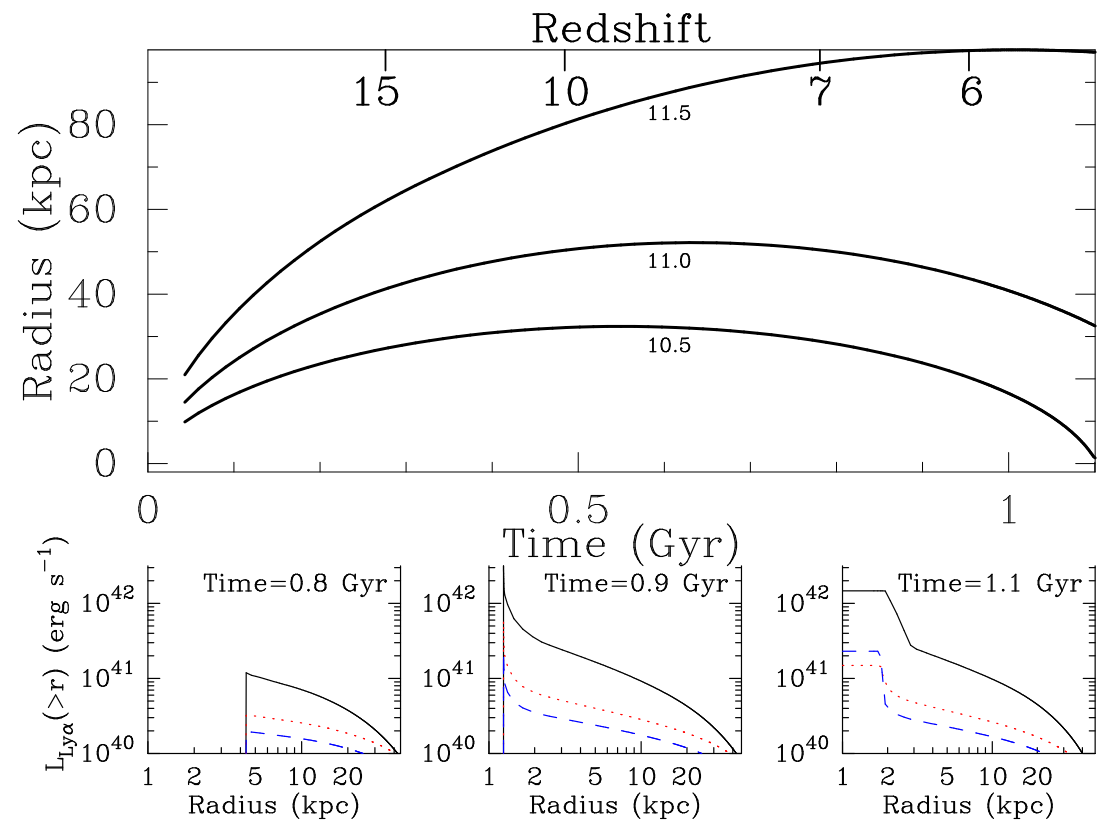

FIG. 1.- The collapse of a $M_{\mathrm{tot}}=10^{11.5} M_{\odot}$ halo in a 1-D hydrodynamical simulation and its accompanying Ly $\alpha$ cooling radiation are shown. The top panel shows the radial evolution of mass shells initially enclosing $\log M_{\text {tot }} / M_{\odot}=10.5,11$ and 11.5 (see labels). The lower horizontal axis shows the age of the Universe in Gyr, while the upper shows redshift. The lower three panels show the total Ly $\alpha$ luminosity outside a sphere of radius $R$ as a function of $R$ (see text for a justification of this peculiar choice) at three times. The total Ly $\alpha$ luminosity derived from the 1-D code is likely underestimated (see text). For comparison, we also show the luminosity in recombination (dotted line) and bremsstrahlung (dashed line) cooling radiation. These processes are clearly subdominant. In the early stages of the collapse (lower left panel) shells at all radii contribute to the total Ly $\alpha$ luminosity. However, during later stages in the collapse the Ly $\alpha$ luminosity is dominated by emission from the central few kpc. This Figure illustrates a property of cooling radiation that is not unique to this spherical collapse model: namely that the total Lya luminosity of a cooling cloud may be dominated by emission from the central few kpc.

\section{COMPACT LY $\alpha$ COOLING RADIATION}

We use a modified version of a one-dimensional (spherically symmetric) hydrodynamical code originally written by Thoul \& Weinberg (1995). For a detailed description of the code the reader is referred to Thoul \& Weinberg (1995). The modifications are described in Dijkstra et al. (2004). The goal of this section is to emphasis the possibility that the Ly $\alpha$ luminosity of compact cooling clouds is comparable to that of star forming galaxies.

We focus on one particular simulation in which a dark matter halo of mass $\log M_{\mathrm{tot}} / M_{\odot}=11.5$ collapses at $z_{c}=4.5$. Such an object is expected to have a Ly $\alpha$ luminosity of $L_{\alpha} \sim$ a few $10^{42}$ $\mathrm{erg} \mathrm{s}^{-1}$ (Haiman et al. 2000), which is within the range of luminosities that is probed by existing Ly $\alpha$ surveys (e.g. Ouchi et al. 2008a). At redshift $z=4.5$ the Universe was $\sim 1.4$ Gyr old. We take snap shots of the simulation at intervals of $75 \mathrm{Myr}$ and read out quantities such as gas density, velocity, cooling rates etc.

The top panel of Figure 1 shows the radial evolution of mass shells initially enclosing $\log M_{\text {tot }} / M_{\odot}=10.5,11$ and 11.5 (see labels). The lower horizontal axis shows the age of the Universe in Gyr, while the upper shows redshift. Each shell initially expands with the Hubble flow and is subsequently decelerated by the enclosed mass until it collapses. The lower three panels show the total Ly $\alpha$ luminosity outside a sphere of radius $R$ as a function of $R$, i.e. $L_{\mathrm{Ly} \alpha}(R)=4 \pi \int_{R}^{\infty} r^{2} d r j_{\alpha}(r)$. The reason for plotting this quantity is that the Ly $\alpha$ volume emissivity, $j_{\alpha}(r)$, increases rapidly toward smaller radii. This causes the total Ly $\alpha$ luminosity emitted by material enclosed within radius $R$ (which is $4 \pi \int_{r_{\min }}^{R} r^{2} d r j_{\alpha}(r)$ ) to depend strongly on the choice of $r_{\min }$. Furthermore, $j_{\alpha}(r)$ is highly uncertain at small radii, because various effects that are not included in the simulation (e.g. star formation) can affect $j_{\alpha}(r), r_{\min }$ and the gas' central density and velocity field. The Ly $\alpha$ luminosity plotted in Figure 1 is only affected at the smallest radii by these uncertainties.

The lower left panel of Figure 1 shows that all radii contribute to the total Ly $\alpha$ luminosity during the early stages of the collapse. However, at later times the density in the inner few kpc builds up (e.g. at time $=1.1 \mathrm{Gyr}$, the number density of hydrogen nuclei is $\gtrsim 0.01 \mathrm{~cm}^{-3}$ for $\mathrm{r} \lesssim 4 \mathrm{kpc}$, see Appendix A which boosts the collisional excitation rate of the Ly $\alpha$ transition. This causes the central few kpc of the collapsing gas to dominate the total Lya cooling luminosity.

Of course, the results presented above are suspicious as they were obtained from a spherically symmetric simulation. In a scenario of hierarchical structure formation halos are build up of mergers of smaller halos that collapsed at earlier times. Gas accretion is therefore likely to occur in dense clumps, which affects the results we obtained above in several ways: (i) Collisions between gas clumps convert the clumps' kinetic energy (due to infall) into thermal energy, which is then radiated away as cooling radiation; (ii) Clumping can boost the the collisional excitation rate of atomic hydrogen and hence the Ly $\alpha$ emissivity.

Only (i) affects the predicted surface brightness profile: In the 1-D calculation, gravitational binding energy is mainly converted into kinetic energy of the infalling gas. This kinetic energy must be (partly) released as cooling radiation in the center of the halo (see Birnboim \& Dekel 2003; Kereš et al. 2005, for more detailed discussions on this topic). Therefore, most of the gravitational binding energy can only be released in the center, which causes cooling radiation emerging from a 1-D simulation to peak too much toward the center. However, Haiman et al. (2000) showed that clumpy accretion also results in surface brightness profile that is peaked towards the center, and that 
the inner $10 \%$ of the gas (in radius) emits $\sim 30 \%$ of total Ly $\alpha$ luminosity. In the calculations of Haiman et al. (2000) gravitational binding energy is emitted continuously while the gas streams towards the center of the halo. Furthermore, 3-D simulations by Kereš et al. (2005) have shown that the gas' infall speed increases down to $\sim 0.5$ virial radii after which the infall velocity slowly decreases (also see Wise \& Abel 2007). In this case gravitational potential energy is radiated away in a more compact region than that described by Haiman et al. (2000). Therefore, we conclude that the actual compactness of Ly $\alpha$ cooling radiation is probably bracketed by the 1-D simulation shown above and the analytic estimate presented by Haiman et al. (2000).

The lack of clumps causes the total Ly $\alpha$ luminosities shown in Figure 1 to be lower than the estimates presented by Haiman et al. (2000), which only depended only on the energy budget of the gas. Haiman et al. (2000) found that the total Ly $\alpha$ luminosity due to cooling can be as large as $10^{43}-10^{44} \mathrm{erg} \mathrm{s}^{-1}$ in halos in the mass range $\log M_{\text {tot }} / M_{\odot}=11-12$. The compactness of the emission implies that a cooling blob $\lesssim 1$ arcsec in diameter may emit Ly $\alpha$ luminosities exceeding $10^{43} \mathrm{erg} \mathrm{s}^{-1}$. This Ly $\alpha$ luminosity is comparable to that observed high-redshift $\operatorname{Ly} \alpha$ emitting galaxies (e.g. Kashikawa et al. 2006). It is worth pointing out that resonant scattering of $\operatorname{Ly} \alpha$ is not expected to flatten the observed surface brightness profile significantly (Dijkstra et al. 2006, hence these source also appear compact on the sky).

It should be pointed out that in the hierarchical structure formation model, the progenitor halos that merge into the halo of interest, are likely substantially more massive that the minimum halo mass that is capable of cooling gas by collisionally exciting atomic transitions (i.e. halos with $T_{\text {vir }} \geq 10^{4} \mathrm{~K}$ which corresponds to $\left.M_{\text {tot }} \gtrsim 10^{8} M_{\odot}\right)$. Hence, gas inside these halos were likely capable of collapsing and forming stars. It is therefore plausible that previously formed stars already exist in the newly formed halo in which gas still needs to cool and collapse to its center. These older stars however are not expected to contribute to the rest-frame UV (and $\operatorname{Ly} \alpha$ ) luminosity of the cloud.

\section{COOLING RADIATION REDWARD OF LY $\alpha$}

In the previous section we concluded that the Ly $\alpha$ luminosity associated with compact cooling gas clouds can be comparable to that of star forming galaxies. In this section we calculate the spectral energy distribution of cooling radiation at wavelengths other than $\operatorname{Ly} \alpha$, and investigate whether this can distinguish a cooling cloud from a star forming galaxy.

\subsection{The 2- $\gamma$ Continuum: Luminosity \& Spectrum}

Yang et al. (2006) found that gas in their simulations becomes self-shielding for densities exceeding $n \gtrsim 200 \bar{n}$, where $\bar{n}$ is the mean cosmic number density of hydrogen (see their Fig 2). In the absence of a photoionizing background, collisional excitation of hydrogen dominates the total cooling rate for gas temperatures of $\sim 1-3 \times 10^{4} \mathrm{~K}$ (see e.g Fig 1 of Thoul \& Weinberg 1995). For higher temperatures the residual neutral hydrogen fraction becomes too small $\left(x_{\mathrm{HI}} \lesssim 1 \times 10^{-3}\right.$ assuming collisional ionization equilibrium, see e.g. House 1964, Hui \& Gnedin 1997), and this cooling mechanism is shut off. Even in the 1D simulation used in $\S[$, which did not include self-shielding, collisional excitation of hydrogen dominates the cooling rate at radii $\lesssim 10 \mathrm{kpc}$ (see Fig 1 ).

Collisions between electrons and neutral hydrogen atoms mainly excite the $n=2$ states (collisional excitation of the $n=3$ states

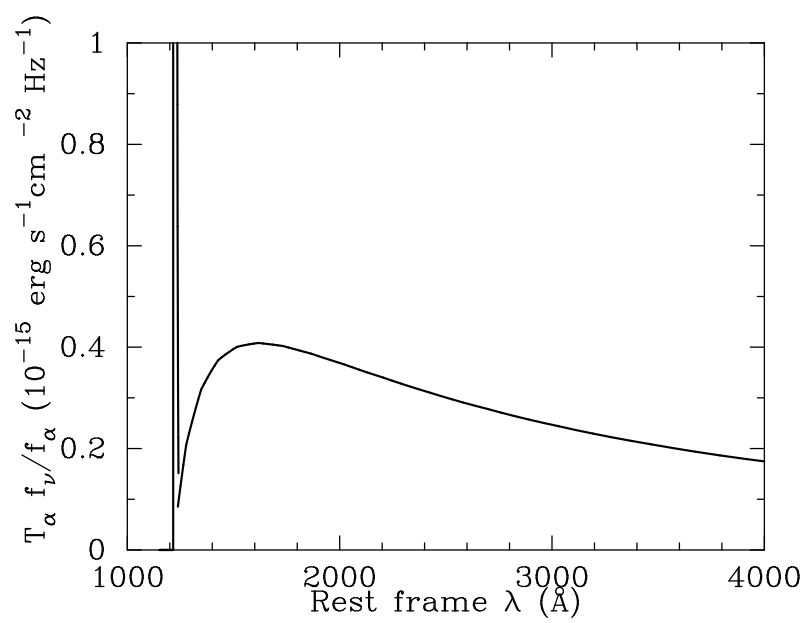

FIG. 2.- Spectral energy distribution of cooling radiation emitted by gas at $T \sim 2 \times 10^{4} \mathrm{~K}$ is shown. A prominent Ly $\alpha$ line (see Fig 3 the width and height of the line shown here are schematic.) is accompanied by a continuum redward of Ly $\alpha$. This continuum is dominated by two-photon transitions from $2 s \rightarrow 1 s$ following collisional excitation of the $2 \mathrm{~s}$ state of atomic hydrogen. For comparison, an unreddened starburst is expected to emit a spectrum of the form $f_{\nu}=$ constant. The units of $f_{\nu}$ are expressed in terms of the observed Ly $\alpha$ line flux (see text).

occurs $\gtrsim 10$ times less frequently at the temperatures considered here). The collisional excitation rates from $1 \mathrm{~s} \rightarrow 2 \mathrm{p}$ and $1 \mathrm{~s} \rightarrow 2 \mathrm{~s}$ are comparable as the effective collision strengths are comparable, $\Omega(1 \mathrm{~s}, 2 \mathrm{~s}) / \Omega(1 \mathrm{~s}, 2 \mathrm{p})=0.6-0.5$ for $T_{\text {gas }}=1-2 \times$ $10^{4} \mathrm{~K}$ (Osterbrock \& Ferland 2006, Table 3.16, the temperature dependence of this ratio is weak 3 . Transitions from $2 \mathrm{~s} \rightarrow 1 \mathrm{~s}$ may only take place when two photons are emitted that have a combined energy of $10.2 \mathrm{eV}$ (Breit \& Teller 1940). This twophoton process results in the following spectrum

$$
f_{2 \gamma}(\nu) d \nu=\frac{\Omega(1 \mathrm{~s}, 2 \mathrm{~s})}{\Omega(1 \mathrm{~s}, 2 \mathrm{p})} \dot{N}_{\mathrm{Ly} \alpha} h \nu \phi\left(\nu / \nu_{\alpha}\right) d \nu / \nu_{\alpha},
$$

where $\dot{N}_{\text {Ly } \alpha}$ is the rate at which Ly $\alpha$ photons are emitted, $\phi\left(\nu / \nu_{\alpha}\right)$ $d \nu / \nu_{\alpha}$ is the probability that 1 photon is emitted in the frequency range $\nu \pm d \nu / 2$, which can be found in Spitzer \& Greenstein (1951). The spectral energy distribution according to Eq 1 is shown in Figure2. The units of $f_{\nu}$ are chosen such that $\int d \nu f_{2 \gamma}(\nu)=$ $[\Omega(1 \mathrm{~s}, 2 \mathrm{~s}) / \Omega(1 \mathrm{~s}, 2 \mathrm{p})] f_{\alpha} / \mathcal{T}_{\alpha}$. Here, $f_{\alpha}$ is the observed Ly $\alpha$ flux and, and $\mathcal{T}_{\alpha}$ is the fraction of $\mathrm{Ly} \alpha$ photons that is transmitted to the observer through the IGM (see below). For example, Figure 2 shows that the peak flux density in the two-photon continuum $f_{2 \gamma, \max }=2 \times 10^{-32} f_{\alpha, 17}\left(0.2 / \mathcal{T}_{\alpha}\right) \mathrm{erg} \mathrm{s}^{-1} \mathrm{~cm}^{-2} \mathrm{~Hz}^{-1}$, where $f_{\alpha} \equiv f_{\alpha, 17} \times 10^{-17} \mathrm{erg} \mathrm{s}^{-1} \mathrm{~cm}^{-2}$. Also shown is a schematic representation of the Ly $\alpha$ line (its shape is arbitrary).

Other process which contribute to wavelengths redward of Ly $\alpha$ include recombination and bremsstrahlung cooling. As already mentioned however, in cooling gas at $T \lesssim 3 \times 10^{4} \mathrm{~K}$ both of these processes are negligible compared to collisional excitation of hydrogen (see $\$ 3.2$ for a more detailed discussion), and their contribution to the continuum spectrum can be safely ignored.

The prominence of the $\operatorname{Ly} \alpha$ line relative to its continuum is

${ }^{3}$ Collisional deexcitation of the 2 s state suppresses the $2-\gamma$ emissivity by a factor of $\left(1+n_{\mathrm{p}} / n_{c}\right)^{-1}$, where $n_{c}=1.5 \times 10^{4} \mathrm{~cm}^{-3}$ is the critical density of the $2 s \rightarrow$ $1 s$ transition, and $n_{p}$ is the number density of protons Osterbrock \& Ferland 2006). Therefore, only gas with densities exceeding $n_{c}$ is expected to emit the two-photon continuum at levels less than that calculated above. These high densities are never reached in the simulation (see Appendix $\mathrm{A}$ and collisional deexcitation is not important in the context of this paper. 


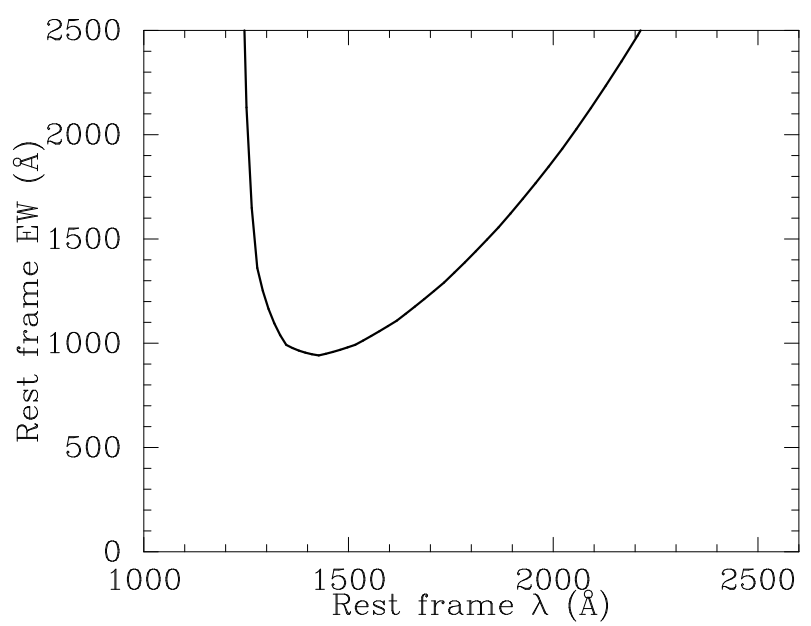

FIG. 3.- The emitted rest frame Ly $\alpha$ equivalent width (EW) of cooling radiation as a function of the wavelength (rest frame) at which the continuum is measured. The EW diverges when $\lambda \rightarrow 1216 \AA$, as the continuum flux density approaches zero. Note that the observed rest frame EW is likely to be a factor of $\sim 5-10$ lower because of scattering of Ly $\alpha$ photons in the IGM (see text).

quantified by the equivalent width (EW) which is defined as $\mathrm{EW} \equiv L_{\alpha} / f_{\lambda}$. Because of the strong wavelength dependence of the continuum between $\lambda=1216-1600 \AA$, the precise EW depends on the wavelength at which the continuum flux density is measured. This is shown in Figure 3, which shows the emitted rest frame Ly $\alpha \mathrm{EW}$ of cooling radiation as a function of the wavelength (rest frame) at which it is measured. The EW diverges when $\lambda \rightarrow 1216 \AA$, as the continuum flux density approaches zero. For $\lambda=1300-1800 \AA$ (rest-frame) the emitted EW is $\sim 1000-1300 \AA$. These EWs are comparable to those emitted by young metal-poor galaxies $\AA$ (Schaerer 2003).

The observed rest-frame EW is less than that calculated above as residual neutral hydrogen in the intergalactic medium (IGM) is expected to scatter a significant fraction of the emitted Ly $\alpha$ flux out of our line of sight. Dijkstra et al. (2007) calculated that only $\mathcal{T}_{\alpha} \sim 0.1-0.3$ of all Ly $\alpha$ that is emitted by high-redshift galaxies is actually detected $\left(\mathcal{T}_{\alpha}=0.2\right.$ was quoted earlier since it corresponds to the median of this range). Moreover, Ly $\alpha$ that emerges from the central collapsing clump is likely to be somewhat blueshifted $(\$ 1)$, which results in a preferred lower value of $\mathcal{T}_{\alpha}$ (Dijkstra et al. 2007). We conclude that the IGM transmission is very likely $\mathcal{T}_{\alpha} \lesssim 0.3$, and that consequently the observed rest-frame $\mathrm{Ly} \alpha \mathrm{EW}$ of cooling radiation is $\mathrm{EW} \lesssim 400$ A.

The observed rest-frame EW among high redshift Ly $\alpha$ emitting galaxies can be EW 100-500 А (e.g. Dawson et al. 2004; Shimasaku et al. 2006). The existence of these large EW Ly $\alpha$ emitting galaxies has led to suggestions that primordial star formation may have been observed (e.g. Malhotra \& Rhoads 2002; Dijkstra \& Wyithe 2007). Indeed, the results presented above suggest that some of these galaxies may be powered by cooling radiation. Figure 2 shows that the spectrum of cooling radiation redward of $\operatorname{Ly} \alpha$ peaks around $\lambda \sim 1600 \AA$, with a sharp drop down to $\lambda=1216 \AA$. For comparison, a powerlaw spectrum of the form $f_{\lambda} \propto \lambda^{-2}$ (or $f_{\nu}=$ constant) is expected from an unreddened starburst and consistent with observations of 27 candidate $z=6$ galaxies in the Hubble Ultra Deep Field (Stanway et al. 2005). If detected, its continuum spec- trum could therefore distinguish cooling clouds from young star forming galaxies. This prospect is explored in more detail below.

\subsection{Prospects for Using 2- $\gamma$ Emission as a Tracer of Cooling Clouds}

\subsubsection{Cooling Clouds and the 2- $\gamma$ Continuum}

The fate of the gas as it approaches the center of the dark matter halo is not known (see Kereš et al. 2005, for more discussion on this). The gas may be slowed down gradually, or may smoothly join a rotating gaseous disk that formed in the center of the dark matter halo. In both these scenarios the gas would remain cold and would emit the spectrum shown in Figure 2 .

However, this is not the case when gas approaches $r=0$ with velocities of order $\sim 100 \mathrm{~km} \mathrm{~s}^{-1}$ (as was the case in the 1-D simulation used in $\S 2$ ). When the gas crashes into the center, it is shock-heated to $T \sim 10^{6} \mathrm{~K}$ and emits bremsstrahlung up to energies of $\sim 100 \mathrm{eV}$. This ionizing radiation creates an $\mathrm{H} \mathrm{II}$ region of $\lesssim$ a few kpc because of the large gas densities in the center of the halo (Birnboim \& Dekel 2003). This ionizing radiation could furthermore doubly ionize helium.

This recombining gas may emit a spectrum that is very similar to that shown in Figure 2. In Figure 4 the solid line shows the spectrum emitted by gas assuming case-A recombination and a gas temperature of $\mathrm{T}=2 \times 10^{4} \mathrm{~K}$. In this case the bound-free (free-free) emissivity is given by $\epsilon=5.3(2.6) \times 10^{-25} \mathrm{erg} \mathrm{s}^{-1}$ $\mathrm{cm}^{3}$ (e.g. Osterbrock \& Ferland 2006). Furthermore, the total emissivity in the two-photon continuum is $\epsilon \sim 5.0 \times 10^{-25} \mathrm{erg}$ $\mathrm{s}^{-1} \mathrm{~cm}^{3}$ (Osterbrock \& Ferland 2006, Table 4.1, and we used that $\epsilon_{2 \gamma} \sim 0.5 \epsilon_{\mathrm{Ly} \alpha}$ ). It is noted that when helium is doubly ionized inside the $\mathrm{H}$ II region, and/or for higher gas temperatures, more continuum emission is emitted at $\lambda<2000 \AA$ (as well as a He II H $\alpha$ line at $\lambda=1640 \AA$ ), which would weaken the appearance of the dip. The dotted line shows the spectrum resulting from bound-bound transitions only (which corresponds exactly to the case shown in Fig 2). Technically the Ly $\alpha+2-\gamma$ radiation is not cooling radiation itself, but is emitted by the cooling cloud as a by-product of recombination cooling radiation (see $\S 10$. Clearly, recombining gas at $T \lesssim 2 \times 10^{4} \mathrm{~K}$ emits a spectrum of similar to that of 'pure' cooling radiation, especially at wavelengths where the spectrum differs from that emitted by star forming galaxies, $\lambda=1200-2000 \AA$.

\subsubsection{Star Forming Galaxies and the 2- $\gamma$ Continuum}

Of course, recombining H II regions (of primordial composition) surrounding stars or quasars emit the same spectrum as that shown in Fig 4 However, in observed galaxies this nebular emission is subdominant to the continuum radiation from stars/quasars, which does not exhibit the dip shown in Figure 4. A notable exception is discussed by Schaerer (2002), who shows that nebular emission dominates the stellar continuum at wavelengths redward of Ly $\alpha$ during the first 1-2 Myr in metal-free galaxies that are forming stars according to topheavy initial mass function (IMF). Hence, these primordial star forming galaxies also exhibit the two-photon dip in their spectra during the first 1-2 Myr of their lifetime (see Fig 5 of Schaerer 2002). On the other hand, these galaxies also emit He III recombination lines such as the He 1640 line, which is not the case for cooling radiation when the gas does not get shock heated to $T \gtrsim 10^{5} \mathrm{~K}$ (as was discussed above in $\S 3.2 .1$ ). 


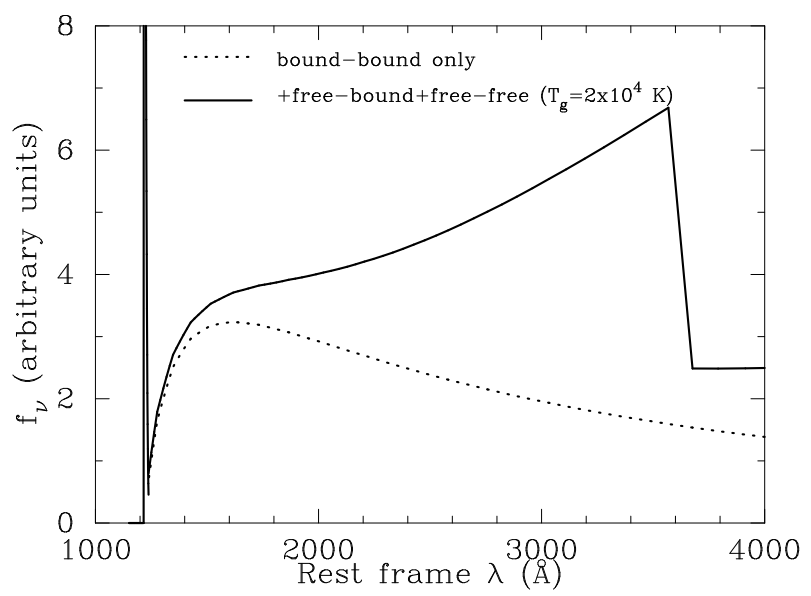

FIG. 4.- The spectrum emitted by recombining gas at $\mathrm{T}=2 \times 10^{4} \mathrm{~K}$ is shown (solid line). This spectrum includes the contribution from free-free, free-bound and bound-bound transitions. The dotted line shows the contribution from bound-bound transitions only, and this spectrum is identical to that of cooling gas (Fig 2). Clearly, the spectra of recombining and cooling gas are very similar at $\lambda \lesssim 2000 \AA$.

\subsubsection{The Impact of Lyo Radiative Transfer}

It is worth pointing out is that for sufficiently large columns of neutral hydrogen gas $\left(N_{\mathrm{HI}} \gtrsim 10^{22} \mathrm{~cm}^{-2}\right)$, Ly $\alpha$ photons scatter so frequently that collisional deexcitation from the $2 p$ level may become important, and the Ly $\alpha$ cooling channel may be suppressed strongly. This process has been studied in more detail by Spaans \& Silk (2006). However, this mechanism only becomes efficient when densities exceed $\gtrsim 10^{2} \mathrm{~cm}^{-3}$, which is much larger than the highest densities in the simulation shown in Figure 11 (see Fig 5 in Appendix 5). Furthermore, this effect enhances the expected 2- $\gamma$ continuum relative to the Ly $\alpha$ line, and if $\operatorname{Ly} \alpha$ were ever detected from these ultracompact cooling cores, then the associated 2- $\gamma$ flux is relatively brighter and thus easier to detect.

So far, the role of dust in the Ly $\alpha$ radiative transfer has not been discussed. One might expect little dust to exist in gas that is still cooling to form stars. However, as mentioned in $\S 2$ in models of hierarchical structure formation, star formation is expected to have occurred in the less massive progenitor halos which merged into the halo of interest. Thus, dust is expected to exist in the cooling cloud. Dust would redden and suppress the UV-continuum, but to quantify this effect theoretically is difficult. Empirically, Bouwens et al. (2006) found the rest-frame UV continuum of $z=6$ Lyman Break Galaxies (LBGs) to be suppressed by dust by a factor of only $\sim 1.4$. It is reasonably to expect the progenitor halos not to contain more dust than these more massive -and hence probably more evolved- LBGs, and we therefore do not expect dust attenuation of continuum cooling radiation to be significant 4 .

The impact of dust on Ly $\alpha$ is less clear. In principle, resonant scattering enhances the total distance $\operatorname{Ly} \alpha$ photons travel through a medium, which makes them more prone to destruction by dust. In this case, the $2-\gamma$ continuum would be enhanced relative to $\operatorname{Ly} \alpha$ and would therefore be easier to detect. On the other hand, Ly $\alpha$ may preferentially avoid destruction by dust

${ }^{4}$ Gnedin et al. (2008) found the mean escape fraction of ionizing radiation in (adaptive mesh refinement) hydrodynamical simulations of high-redshift galaxies to be $\left\langle f_{\text {esc }}\right\rangle \sim 1 \%$. However, the opacity to ionizing photons in these simulations was found to be dominated by neutral atomic hydrogen and helium. in a two-phase medium in which dust resides in cold $\left(T \sim 10^{4}\right.$ $\mathrm{K})$ neutral denser clumps embedded in a hot medium (Neufeld 1991; Hansen \& Oh 2006). In this scenario, the 2- $\gamma$ continuum would be attenuated (by a factor of $\lesssim 1.4$ as we argued above), but the $\mathrm{Ly} \alpha$ would be freely transmitted. It is therefore good to keep in mind that our quoted $\operatorname{Ly} \alpha$ luminosities have not corrected for dust attenuation, but that this correction is not necessarily needed.

\section{DISCUSSION \& CONCLUSIONS}

The collapse and condensation of baryons into the center of their host dark matter halos is accompanied by emission of radiation that may be detectable as compact UV-continuum and Ly $\alpha$ emission with Ly $\alpha$ luminosities as high as $\sim 10^{42}-10^{43}$ $\mathrm{erg} \mathrm{s}^{-1}$ in halos of mass $M=10^{11}-10^{12} M_{\odot}$. These luminosities are comparable to those detected in narrowband surveys for redshifted Ly $\alpha$ emission.

Cooling clouds emit continuum radiation redward of $\operatorname{Ly} \alpha$ that is dominated by two-photon transitions from $2 \mathrm{~s} \rightarrow 1 \mathrm{~s}$. The emitted Ly $\alpha$ equivalent width (EW) relative to this continuum is $\mathrm{EW} \sim 1000-1300 \AA$ (restframe), which is comparable to the EW emitted by galaxies forming either metal-poor or very massive stars. The IGM is likely to transmit only $\lesssim 30 \%$ of this Ly $\alpha$ flux and the observed rest frame equivalent width is therefore EW $\lesssim 400 \AA$. This is comparable to the observed equivalent widths among high-redshift Ly $\alpha$ emitting galaxies. It is therefore possible that existing surveys have detected compact Ly $\alpha$ emission that was emitted by cooling clouds.

Because of the dominance of the two-photon continuum, cooling clouds emit a spectrum that peaks at $\lambda \sim 1600 \AA$ (rest-frame), and sharply drops down to $\lambda=1216 \AA$. This peculiar spectral shape distinguishes cooling clouds from observed young star forming galaxies, whose continuum is dominated by radiation from stars/quasars (e.g. Stanway et al. 2005). However, this is not true for metal-free galaxies that are forming stars according to top-heavy initial mass function. In these primordial galaxies, nebular emission may dominate the continuum redward of Ly $\alpha$ during the first few Myr of their lifetime (Schaerer 2002). On the other hand, these galaxies also emit He III recombination lines such as the He 1640 line, which is not the case for cooling radiation when the gas is not shock heated to $T \gtrsim 10^{4} \mathrm{~K}$ (see § 3.2). Furthermore, deeper Ly $\alpha$ observations of compact cooling clouds should find weaker emission on larger scales. However, the same applies to $\operatorname{Ly} \alpha$ emitting star forming galaxies, which are likely surrounded by faint Ly $\alpha$ halos due to resonant scattering in the surrounding IGM (e.g. Loeb \& Rybicki 1999; (Dijkstra \& Loeb 2008). This scattered Ly $\alpha$ emission is not accompanied by continuum radiation redward of the Ly $\alpha$ line, which could distinguish it from spatially extended Ly $\alpha$ cooling radiation (polarimetry may provide additional insights, see Dijkstra \& Loeb 2008).

Ongoing and future surveys for redshifted Ly $\alpha$ emission aim to detect Ly $\alpha$ emitters out to redshifts as high as $z \sim 6-12$ (e.g. Stark et al. 2007; Nilsson et al. 2007, and references therein). These observations will provide new insights into the epoch of reionization and galaxy formation at high redshift. Primordial galaxies -like cooling clouds- may emit very large EW Ly $\alpha$ emission lines (e.g. Schaerer 2003). In order to measure the EW of the Ly $\alpha$ line and to determine whether primordial galaxies are detected, the continuum redward of Ly $\alpha$ must be detected. This is challenging; if the observed flux in the Ly $\alpha$ line is $f_{\alpha, 17}$ (in units of $10^{-17} \mathrm{erg} \mathrm{s}^{-1} \mathrm{~cm}^{-2}$ ) and its equivalent 
width is EW (in $\AA$ ), then the $\mathrm{AB}$-magnitude of the continuum is $m_{\mathrm{AB}}=30.4-2.5 \log f_{\alpha, 17}+2.5 \log (\mathrm{EW} / 200)$. For comparison, the Hubble Ultra Deep Field reached an $8-\sigma$ limiting magnitude in the $z^{\prime}$ filter of $z_{\mathrm{AB}}^{\prime}=28.5$ (Bunker et al. 2004).

Detecting both the Ly $\alpha$ line and the continuum is not only desirable for identifying primordial galaxies. An additional advantage of combining $\operatorname{Ly} \alpha$ and continuum measurements of high-redshift galaxies is that it can greatly improve the constraints on the epoch of reionization (e.g. Kashikawa et al.2006; McQuinn et al. 2007) over constraints obtained using Ly $\alpha$ alone. The work presented in this paper suggests that if primordial galaxies can be detected and identified, then the same applies to cooling clouds. Moreover, primordial galaxies may only be luminous in Ly $\alpha$ for $\lesssim 10$ Myr (Malhotra \& Rhoads 2002; Dijkstra \& Wyithe 2007). Cooling clouds could be luminous in $\mathrm{Ly} \alpha$ for longer than $10 \mathrm{Myr}$, which would make them more easily detectable than primordial galaxies. The discovery of two-photon dominated Ly $\alpha$ emitters would offer us an exciting glimpse of either the process of gas cooling in newly forming galaxies, or of star formation in the first primordial galaxies.

Acknowledgments I thank Marco Spaans for his comments on an earlier draft of this paper, and Zoltán Haiman for useful discussions. This work was supported by Harvard University funds.

\section{REFERENCES}

Basu-Zych, A., \& Scharf, C. 2004, ApJ, 615, L85

Birnboim, Y., \& Dekel, A. 2003, MNRAS, 345, 349

Bouwens, R. J., Illingworth, G. D., Blakeslee, J. P., \& Franx, M. 2006, ApJ, 653,53

Breit, G., \& Teller, E. 1940, ApJ, 91, 215

Bunker, A. J., Stanway, E. R., Ellis, R. S., \& McMahon, R. G. 2004, MNRAS, 355,374

Chambers, K. C., Miley, G. K., \& van Breugel, W. J. M. 1990, ApJ, 363, 21

Dawson, S., et al. 2004, ApJ, 617, 707

Dey, A., et al. 2005, ApJ, 629, 654

Dijkstra, M., Haiman, Z., Rees, M. J., \& Weinberg, D. H. 2004, ApJ, 601, 666

Dijkstra, M., Haiman, Z., \& Spaans, M. 2006, ApJ, 649, 14

Dijkstra, M., Lidz, A., \& Wyithe, J. S. B. 2007, MNRAS, 377, 1175

Dijkstra, M., \& Wyithe, J. S. B. 2007, MNRAS, 379, 1589

Dijkstra, M., \& Loeb, A. 2008, MNRAS, 386, 492

Fardal, M. A., Katz, N., Gardner, J. P., Hernquist, L., Weinberg, D. H., \& Davé, R. 2001, ApJ, 562, 605

Gnedin, N. Y., Kravtsov, A. V., \& Chen, H.-W. 2008, ApJ, 672, 765

Haiman, Z., Spaans, M., \& Quataert, E. 2000, ApJ, 537, L5

Hansen, M., \& Oh, S. P. 2006, MNRAS, 367, 979

House, L. L. 1964, ApJS, 8, 307

Hui, L., \& Gnedin, N. Y. 1997, MNRAS, 292, 27

Kashikawa, N., et al. 2006, ApJ, 648, 7

Kereš, D., Katz, N., Weinberg, D. H., \& Davé, R. 2005, MNRAS, 363, 2

Loeb, A., \& Rybicki, G. B. 1999, ApJ, 524, 527

Malhotra, S., \& Rhoads, J. E. 2002, ApJ, 565, L71

Matsuda, Y., et al. 2004, AJ, 128, 569

Matsuda, Y., Yamada, T., Hayashino, T., Yamauchi, R., \& Nakamura, Y. 2006, ApJ, 640, L123

Matsuda, Y., Iono, D., Ohta, K., Yamada, T., Kawabe, R., Hayashino, T., Peck, A. B., \& Petitpas, G. R. 2007, ApJ, 667, 667

McQuinn, M., Lidz, A., Zahn, O., Dutta, S., Hernquist, L., \& Zaldarriaga, M. 2007, MNRAS, 377, 1043

Mori, M., Umemura, M., \& Ferrara, A. 2004, ApJ, 613, L97

Neufeld, D. A. 1991, ApJ, 370, L85

Nilsson, K. K., Fynbo, J. P. U., Møller, P., Sommer-Larsen, J., \& Ledoux, C. 2006, A\&A, 452, L23

Nilsson, K. K., Orsi, A., Lacey, C. G., Baugh, C. M., \& Thommes, E. 2007, A\&A, 474, 385

Osterbrock, D. E., \& Ferland, G. J. 2006, Astrophysics of gaseous nebulae and active galactic nuclei, 2nd. ed. by D.E. Osterbrock and G.J. Ferland. Sausalito, CA: University Science Books, 2006,

Ouchi, M., et al. 2008a, ApJS, 176, 301

Ouchi, M., et al. 2008b, ArXiv e-prints, 807, arXiv:0807.4174

Saito, T., Shimasaku, K., Okamura, S., Ouchi, M., Akiyama, M., \& Yoshida, M. 2006, ApJ, 648, 54
Saito, T., Shimasaku, K., Okamura, S., Ouchi, M., Akiyama, M., Yoshida, M., \& Ueda, Y. 2007, ArXiv e-prints, 705, arXiv:0705.1494

Schaerer, D. 2002, A\&A, 382, 28

Schaerer, D. 2003, A\&A, 397, 527

Shimasaku, K., et al. 2006, PASJ, 58, 313

Smith, D. J. B., \& Jarvis, M. J. 2007, MNRAS, 378, L49

Spaans, M., \& Silk, J. 2006, ApJ, 652, 902

Spergel, D. N., et al. 2007, ApJS, 170, 377

Spitzer, L. J., \& Greenstein, J. L. 1951, ApJ, 114, 407

Stanway, E. R., McMahon, R. G., \& Bunker, A. J. 2005, MNRAS, 359, 1184

Stark, D. P., Ellis, R. S., Richard, J., Kneib, J.-P., Smith, G. P., \& Santos, M. R. 2007, ApJ, 663, 10

Stark, D. P., Loeb, A., \& Ellis, R. S. 2007, ApJ, 668, 627

Steidel, C. C., Adelberger, K. L., Shapley, A. E., Pettini, M., Dickinson, M., \& Giavalisco, M. 2000, ApJ, 532, 170

Taniguchi, Y., \& Shioya, Y. 2000, ApJ, 532, L13

Thoul, A. A. \& Weinberg, D. H. 1995, ApJ, 442, 480

Villar-Martín, M. 2007, New Astronomy Review, 51, 194

Wise, J. H., \& Abel, T. 2007, ApJ, 665, 899

Yang, Y., Zabludoff, A. I., Davé, R., Eisenstein, D. J., Pinto, P. A., Katz, N., Weinberg, D. H., \& Barton, E. J. 2006, ApJ, 640, 539

\section{APPENDIX A}

\section{OUTPUT FROM THE SIMULATION}

Two snapshots of the simulation used in $\S 2$ are shown in Figure 5. The radial dependence of the number density of $\mathrm{H}$-nuclei $\left(n_{\mathrm{H}}\right.$, upper panel), the fraction of neutral atomic hydrogen gas $\left(x_{\mathrm{HI}}=n_{\mathrm{HI}} / n_{\mathrm{H}}\right.$, central panel $)$, and the gas temperature $(\mathrm{T}$, lower panels) are shown at time $=0.9 \mathrm{Gyr}$ (solid lines) and time $=1.1$ Gyr (dashed lines). Snapshots of the simulation at these times were also shown in Figure 1 .

Figure 5 shows that the gas temperature in the inner $10 \mathrm{kpc}$ is $T_{\text {gas }} \lesssim 3 \times 10^{4} \mathrm{~K}$ at both times, which corresponds to the temperature regime where collisional excitation of hydrogen dominates gas cooling. Note that the snapshot at time $=1.1 \mathrm{Gyr}$ shows some strange behaviour at $r \lesssim 2 \mathrm{kpc}$. This is related to the formation of a shock that is produced by the gas that is radially moving inwards at a supersonic speed, which causes the gas to shock-heat to $T>10^{5} \mathrm{~K}$. As was mentioned in $\S 2$ the simulation results are suspicious in these innermost regions.

Lastly, we point out that the gas density is well below the critical density, $n_{c}=1.5 \times 10^{4} \mathrm{~cm}^{-3}$, at which collisional deexcitation of the $2 \mathrm{~s}$-state becomes important.

\section{APPENDIX B}

\section{LY $\alpha$ RECOMBINATION VS. LY $\alpha$ COOLING RADIATION}

Here we compare the Ly $\alpha$ cooling rate due to collisional excitation, $\Lambda_{\mathrm{Ly} \alpha}$, with the Ly $\alpha$ emission rate due to recombination, $R_{\mathrm{Ly} \alpha}$. Their ratio is given by

$$
\mathcal{R} \equiv \frac{\Lambda_{\mathrm{Ly} \alpha}}{R_{\mathrm{Ly} \alpha}}=\frac{7.3 \times 10^{-19} x_{\mathrm{HI}} \exp \left(\frac{-118400}{T}\right)}{0.68 \alpha_{\mathrm{rec}, \mathrm{B}} \times h \nu_{\alpha} \times\left(1.0-x_{\mathrm{HI}}\right)},
$$

where $\alpha_{\mathrm{rec}, \mathrm{B}}=2.6 \times 10^{-13}\left(T / 10^{4} \mathrm{~K}\right)^{-0.7} \mathrm{~cm}^{3} \mathrm{~s}^{-1}$ is the case- $\mathrm{B}$ recombination coefficient. For $T=3 \times 10^{4} \mathrm{~K}$ we find $\mathcal{R}=1.0 \times$ $\left(x_{\mathrm{HI}} / 10^{-4}\right)$ (for $\left.x_{\mathrm{HI}} \ll 1\right)$, and $\mathcal{R}$ increases with $T$. That is, even in a highly ionized plasma, a significant fraction of the emitted Ly $\alpha$ photons are produced following collisional excitation of the much rarer neutral hydrogen atoms. 


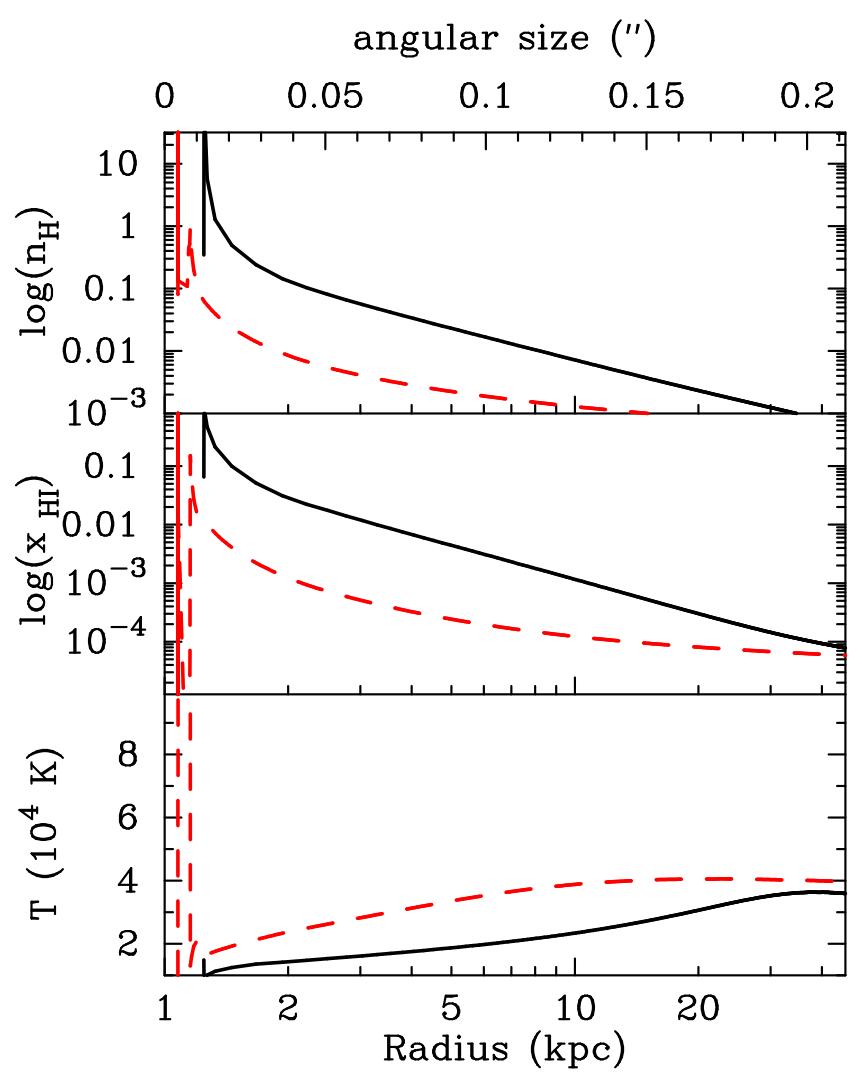

FIG. 5. - Simulation outputs at time $=0.9 \mathrm{Gyr}$ (solid lines $)$ and time $=1.1 \mathrm{Gyr}$ (dashed lines) are shown. The upper/middel/lower panel shows the radial dependence of the gas density/ neutral fraction of atomic hydrogen/temperature.

These quantities were used to compute the cooling rates shown in Figure 1 The snapshot at time $=1.1 \mathrm{Gyr}$ shows that the simulation behaves strangely in the inner $2 \mathrm{kpc}$ (see text). 